\title{
Don't Fuss, Focus: The Mediating Effect of On-Task Thoughts on the Relationship between Error Approach Instructions and Task Performance
}

\author{
Nicoletta G. Dimitrova* and Cathy van Dyck \\ VU University Amsterdam, The Netherlands \\ Edwin A.J. van Hooft \\ University of Amsterdam, The Netherlands \\ Peter Groenewegen \\ VU University Amsterdam, The Netherlands
}

\begin{abstract}
People working on a task can make errors along the way. How people deal with an error, however, depends on the type of error approach they apply. One approach, error management, focuses on increasing the positive and decreasing the negative consequences of errors. A second approach, error prevention, focuses on working faultlessly. In two experiments, we manipulated error approach through task instructions and measured on-task thoughts and offtask thoughts. In Experiment $1(N=78)$, error management resulted in more on-task thoughts, but no differences were found for off-task thoughts. Experiment $2(N=76)$ replicated the findings of Experiment 1, and further demonstrated that error management resulted in better analogical and adaptive transfer performance, and that these effects were mediated by on-task thoughts. Our findings point toward the benefits of error management instructions for people and organisations. Specifically, error management instructions make people more focused on the task during practice, as indicated by on-task thoughts, which in turn results in higher performance after practice.
\end{abstract}

* Address for correspondence: Nicoletta G. Dimitrova, VU University Amsterdam, De Boelelaan 1081, 1081 HV, Amsterdam, The Netherlands. Email: n.g.dimitrova@vu.nl

This article is based on chapter four from Nicoletta Dimitrova's doctoral dissertation. Study 1 was presented at the Academy of Management annual meeting in Montreal, Canada, August 2010. Study 2 was presented at the Academy of Management annual meeting in Boston, Massachusetts, August 2012. The first author thanks Dov Eden, Wolfgang Steinel, and Lindred Greer for their helpful comments on earlier versions of this article and Annemiek van Os and Freek van Berkel for coding the manipulation check segments. We also thank the two anonymous reviewers and the editor for their invaluable assistance in improving the paper. 


\section{INTRODUCTION}

People try to prevent errors because of the negative consequences they may involve, such as stress, frustration, financial loss, and loss of lives (Reason, 1990). Because errors are inevitable (Reason, 1997), it is critical to develop strategies that can help people to continue to pursue a goal after they commit an error. In this way, errors can be effectively corrected, lowering the likelihood of severe negative consequences, and leading to learning from errors, which can eventually affect future task pursuit and result in higher-quality outcomes and better performance (Van Dyck, Frese, Baer, \& Sonnentag, 2005).

Errors are defined as "all those occasions in which a planned sequence of mental or physical activities fails to achieve its intended outcome, and where these failures cannot be attributed to the intervention of some chance agency" (Reason, 1990, p. 9). Errors are characterised by three main features: (1) they are unintentional, (2) they occur only in goal-directed action, and (3) they are potentially avoidable (Frese \& Zapf, 1994; Hofmann \& Frese, 2011; Reason, 1990).

Two main approaches to errors have been previously outlined: error prevention and error management (e.g. Frese \& Zapf, 1994; Hofmann \& Frese, 2011). Although both approaches share the goal of minimising the negative consequences of errors, the way that the two approaches achieve this goal differs. Error prevention entails a relatively negative view of errors and aims to prevent their negative consequences by avoiding errors altogether. Although prevention can be important for reducing errors, it cannot completely eliminate them (Reason, 1997) and it does not prepare people for proper error handling once an error has occurred. In addition, focusing only on error prevention blinds individuals or organisations to the learning potential present in some errors (Sitkin, 1992).

Error management, in contrast, is based on the assumption that error occurrence cannot be completely eradicated and that errors can have both negative and positive consequences. Based on these assumptions, error management proposes that one should try to use errors in a constructive manner by learning from them, as well as by attempting to reduce their negative consequences. Error management thus tries to minimise the negative and maximise the positive consequences of errors.

Research has shown that error management leads to better performance than error prevention for both individuals and organisations (e.g. Keith \& Frese, 2008; Van Dyck et al., 2005). Less is understood, however, about the underlying psychological mechanisms through which error approach affects performance; that is, we are still unclear about why error management is effective. Uncovering the mechanisms through which error approach affects performance is important not only because it helps us understand the 
theoretical underpinnings of the effects of error management on performance, but also because knowing which mechanisms work can help with the implementation of error management in practice.

Researchers have proposed three main mechanisms through which error approach can affect performance: cognitive (e.g. metacognition, Bell \& Kozlowski, 2008; Keith \& Frese, 2005), emotional (e.g. frustration, Debowski, Wood, \& Bandura, 2001; Wood, Kakebeeke, Debowski, \& Frese, 2000; emotion control, Keith \& Frese, 2005; anxiety, Bell \& Kozlowski, 2008), and motivational (intrinsic motivation, Bell \& Kozlowski, 2008; Debowski et al., 2001; Wood et al., 2000; self-efficacy, Bell \& Kozlowski, 2008; Debowski et al., 2001). In the current paper, we investigate how active/ exploratory practice of a novel complex task combined with instructions that either advise people to prevent errors (error prevention) or to make and learn from errors (error management) affect people's cognitions and subsequent performance. Specifically, we are interested in whether providing people with an error management strategy during task practice benefits post-practice performance by positively affecting people's ability to stay on-task and minimise task-undirected (off-task) thoughts.

We extend the work of Keith and Frese (2005), who implied that the allocation of cognitive resources to on-task, off-task thoughts, and selfregulation may mediate the effect of error approach on performance. Although Keith and Frese (2005) established the mediating role of selfregulation (metacognition and emotion control), no study so far has empirically tested on-task and off-task thoughts as mediators. Such a lack of research is surprising as scholars have consistently suggested that the presence of off-task thoughts and the lack of on-task thoughts could negatively affect performance (e.g. Heimbeck, Frese, Sonnentag, \& Keith, 2003; Keith \& Frese, 2005; Nordstrom, Wendland, \& Williams, 1998). Accordingly, our first contribution to the literature is investigating whether the allocation of cognitive resources to on-task and off-task thoughts mediates the effect of error approach on performance.

Our second contribution involves disentangling the role of type of practice and type of instructions. According to the error management training and the active learning literatures (Bell \& Kozlowski, 2008; Hofmann \& Frese, 2011; Keith \& Frese, 2005, 2008), errors during training, as a form of task exploration, are helpful in themselves. We, however, argue that it is not erring itself that helps people when practicing a new task. Rather, how errors are approached makes a difference. By allowing all participants to make errors, we address a limitation of prior work: the confounding of (a) exploratory task practice (practice during which people can make errors) with error management instructions and (b) step-by-step practice (practice during which people cannot make errors) with error prevention instructions (Bell \& Kozlowski, 2008; Keith \& Frese, 2008). 
Our third contribution relates to investigating to what extent even short task practice with minimal error approach instructions can affect performance. We examine whether performance effects reported for extended training sessions in previous research can be generalised to short task instructions. Systems and tasks people face every day increase in complexity, while the time needed for learning how to deal with them diminishes. This creates an interesting paradox in that people are expected to learn more than before in much less time. Because it is not always possible to spend multiple hours on training, optimising by decreasing the amount of time spent on learning, while maintaining positive performance effects, is both important and practically relevant. In contrast to previous research focusing mainly on longer training (e.g. Bell \& Kozlowski, 2008; Dormann \& Frese, 1994; Heimbeck et al., 2003; Joung, Hesketh, \& Neal, 2006; Nordstrom et al., 1998), we examine the effects of brief error management and error prevention instructions during practicing a novel task. Note that we do not define our context as training or error management training per se; rather, our experiments combine brief errorframed (management or prevention) instructions and short task practice.

\section{ON- AND OFF-TASK THOUGHTS}

Making errors is disruptive for goal-directed action and, as such, is likely to affect people's cognitive processing by prompting an individual to stop and think why an error has occurred or what its consequences may be (Ivancic \& Hesketh, 1995/1996). Kanfer and Ackerman's (1989) cognitive resource allocation theory states that people have limited attentional/cognitive resources. These resources can be allocated to on-task thoughts, off-task thoughts, and self-regulation (Kanfer \& Ackerman, 1989). At their core, on-task and offtask thoughts entail attention toward versus away from the task at hand (e.g. Sarason, Sarason, Keefe, Hayes, \& Shearin, 1986). Self-regulation involves (re)directing cognitive resources back to the task when one is experiencing off-task thoughts (Keith \& Frese, 2005).

On-task thoughts can be defined as attention directed towards the task and task completion (e.g. thinking about the rules of the task) or as a form of task-concentration and focused task-engagement (Smallwood, Obonsawin, \& Heim, 2003). On-task thoughts are theoretically and conceptually similar to mental/cognitive focus (Lee, Sheldon, \& Turban, 2003), which indicates the capacity to stay focused on the activity one is currently engaged in. Off-task thoughts, in contrast, involve allocating attention to task-undirected thoughts (e.g. thinking about an appointment later this week). Thus, they involve temporary attentional lapses during which "an individual's attention becomes temporarily disengaged from the task and is instead directed internally within participants" (Smallwood, Riby, Heim, \& Davis, 2006, p. 220). Kanfer and Ackerman's (1989) cognitive resource allocation theory implies 
that on-task thoughts are negatively related to off-task thoughts. Consequently, the more off-task thoughts a person has, the less mental capacity that person would have for on-task thoughts and vice versa. In other words, off-task thoughts compete with on-task thoughts for limited cognitive resources (Sarason et al., 1986).

\section{ERROR APPROACH, ON-TASK THOUGHTS, AND OFF-TASK THOUGHTS}

Making an error during skill acquisition can guide one's thoughts away from the task and in this way jeopardise learning (Joung et al., 2006; Van Der Linden, Sonnentag, Frese, \& Van Dyck, 2001). Research has suggested that suppressing negative thoughts or emotions is more draining than reappraising a negative event (Richards \& Gross, 2000). Thus, suppressing one's thoughts after making an error (e.g. trying not to think that one is performing poorly) is more cognitively costly than reappraising potential error situations beforehand by reframing errors as learning opportunities. We expect that if people apply an error management approach, they will be less prone to direct attention to various possibly disruptive off-task thoughts than if people apply an error prevention approach. Subsequently, error approach is expected to influence the amount of cognitive resources one can spend on task-directed (on-task) thoughts, making people with an error management approach less likely to waste effort on off-task thoughts than people with an error prevention approach. In other words, if people worry less about making errors, because errors are framed positively, they have more cognitive resources available for actively mastering the task. If one cannot reappraise errors as positive learning opportunities, every new error indicates that one is getting farther away from the goal of working faultlessly. As error prevention instructions frame diligence and working faultlessly as beneficial, making an error is expected to result in more negative off-task thoughts and fewer on-task thoughts than error management.

Hypothesis 1: Error management leads to more on-task thoughts than error prevention.

Hypothesis 2: Error management leads to fewer off-task thoughts than error prevention.

\section{PERFORMANCE}

Research has shown that error management leads to better performance than error prevention (Keith \& Frese, 2008) because it increases positive transfer - transferring previously learned knowledge or skills from one task 
to another (Keith \& Frese, 2005). In the current research we examine two types of transfer-related performance: analogical and adaptive.

\section{Analogical Transfer Performance}

Analogical transfer "involves using a familiar problem to solving a problem of the same type" (Ivancic \& Hesketh, 2000, p. 1967). Analogical transfer requires the direct application of a rule, skill, or a procedure that was learned during practice. Encountering errors is expected to sensitise people to similar errors in the future, which leads to an increased capacity to successfully handle those and, as such, promotes analogical transfer (Ivancic \& Hesketh, 2000). In addition, we argue that acquiring the necessary skills during task practice would strongly depend not only on the making of errors per se but also on the framing of errors, which can influence the extent to which people can learn from the errors made.

Research on error approach has found small differences in analogical transfer performance (see Keith \& Frese, 2008). A possible explanation for the small effects may be the coupling of error management instructions with error-based training and error prevention instructions with errorless training, both of which can independently lead to similar levels of analogical transfer (for a discussion see Keith \& Frese, 2008). We wanted to test whether error management instructions would be more beneficial for analogical transfer than error prevention instructions, while allowing all participants to make errors. One can speculate that error management due to its reframing of errors as challenges to master and learn from will help people acquire more information from errors during task practice. Error prevention, in contrast, frames errors as threats to working faultlessly. Because of this, people may want to disengage from the error situation as quickly as possible, and consequently learn less from errors than someone who reappraises errors as learning opportunities.

Hypothesis 3: Error management leads to better analogical transfer performance than error prevention.

\section{Adaptive Transfer Performance}

Adaptive transfer "involves using one's existing knowledge base to change a learned procedure, or to generate a solution to a completely new problem" (Ivancic \& Hesketh, 2000, p. 1968). Adaptive transfer requires people to adapt to a new problem that structurally differs from those previously solved during practice (Ivancic \& Hesketh, 1995/1996). Ford, Smith, Weissbein, Gully, and Salas (1998) explained that for training to be practically useful, it should "prepare individuals to apply what was learned to more complex 
situations than were experienced during training” (p. 220). Error management involves learning how to handle unexpected complications and errors, which makes it an especially effective strategy in instigating adaptive transfer (Keith \& Frese, 2005; 2008). In addition, by reframing errors as learning opportunities, error management stimulates people to pay more attention to the important information errors reveal and thus assists them in developing better mental models of the task (Frese, Brodbeck, Heinbokel, Mooser, Schleiffenbaum, \& Thiemann, 1991).

Research consistently shows that error management has a beneficial effect on adaptive transfer when compared to various alternative methods (e.g. Bell \& Kozlowski, 2008; Dormann \& Frese, 1994; Heimbeck et al., 2003; Keith \& Frese, 2005). It is important, however, to replicate the effect of error management on adaptive transfer performance under conditions of short task practice, to show the expected benefits of error management instructions over error prevention instructions.

Hypothesis 4: Error management leads to better adaptive transfer performance than error prevention.

Note that in our current theorising we differentiate between practice and transfer performance. We only expect error management to lead to improved performance after, but not during, practice itself. Encouraging people to make errors during practice likely results in similar or inferior practice performance of the error management group as compared to the error prevention group. The distinction between performance during training/practice and test/transfer performance is vital because manipulations that positively affect training performance may negatively affect performance after training and vice versa (Keith \& Frese, 2005; Wood et al., 2000). Accordingly, metaanalytic results show that error management is beneficial to transfer rather than to training performance (Keith \& Frese, 2008).

Finally, we hypothesise that the effects of error approach on analogical and adaptive transfer performance are mediated by on-task and off-task thoughts, such that error management results in better analogical and adaptive transfer performance than error prevention because it induces more on-task thoughts and fewer off-task thoughts. Although our predictions regarding mediation by on- and off-task thoughts are theoretically grounded in prior research on error approach (Keith \& Frese, 2005; Joung et al., 2006), the current studies are the first to test whether this mechanism indeed clarifies the effect of error approach on performance. Our conceptual model is outlined in Figure 1.

Hypothesis 5: On-task thoughts mediate the effect of error approach on (a) analogical and (b) adaptive transfer performance.

Hypothesis 6: Off-task thoughts mediate the effect of error approach on (a) analogical and (b) adaptive transfer performance. 


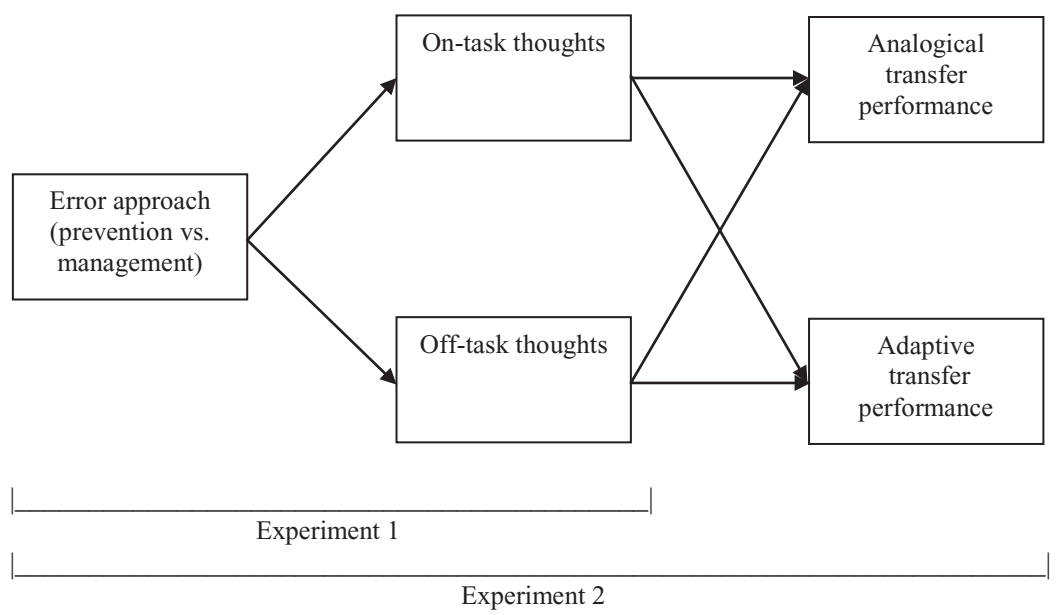

FIGURE 1. Research model.

\section{EXPERIMENT 1}

\section{Method}

Participants and Design. The final sample consisted of 78 students at the university of the first author and one non-student (36 females and 42 males; $M_{\text {age }}=21.05, S D=3.21$ ) who were recruited via flyers advertising the study distributed in the university canteen. All participants were randomly assigned to a two-group between-participant design with error management and error prevention as conditions. All participants received €7 for participation.

The error management group consisted of 24 males and 16 females. The error prevention group consisted of 18 males and 20 females. Five participants were removed from the initial sample: Two due to problems with the procedure during the experiment (one participant took 40 minutes longer to finish the study, one participant responded without reading multiple items) and three participants failed the manipulation check at the end of the study.

Procedure. Participants were seated in separate cubicles and all instructions and questions were presented on a computer screen. The experiment took approximately 60 minutes. We provided a brief description of the purpose of the experiment as an investigation of strategic insight - the type of strategic thinking people use while playing games. 
Manipulation. The error management manipulation was adapted from Keith and Frese (2005), but shortened in a manner consistent with the error management manipulation by Nordstrom et al. (1998). The error prevention manipulation was self-developed, partially based on the error avoidant instructions by Nordstrom et al. (1998). The manipulation texts were comparable in size and wording. Both management and prevention were framed as good for learning and subsequent performance. Participants in the error management condition read that "the occurrence of errors can be very useful because a lot can be learned from errors since they point you towards things you do not know" and that "people who learn how to deal with errors succeed more". In the error prevention condition the text stressed the importance of working without making errors and suggested that "people who learn how to prevent errors succeed more". The text stated that "people who work meticulously and without errors perform better, which is why one should try to think in advance of how to prevent errors". The text also suggested that one can learn a lot if one strives to work without errors. The manipulations were reinforced by presenting three short statements on screen (cf. Keith \& Frese, 2005).

Game Task. We used the game Pingus $\subset$ (Ruhnke, 2010), which is a free-source LemmingsTM-inspired computer game. The game was appropriate in the current context because making errors was highly likely and using either an error management or an error prevention approach was possible. The main objective of the game is to safely lead a group of penguins from the beginning to the end of a level with as small a number of casualties as possible. If not properly guided, the penguins venture into various deadly situations (e.g. jumping off cliffs). In order to make sure a certain percentage of penguins reaches the end of a level, the player must develop strategies by using a set of functions that can be distributed to individual penguins.

Following the experimental manipulation, participants were introduced to the goal of the game and to the game controls. After reading the instructions, participants practiced the game for 25 minutes. During the allotted time participants finished as many levels as possible. Everyone started from the same basic level and could not go on to the next one until the current level was completed successfully.

Dependent Variables. After playing the game for 25 minutes, participants filled in the measures of on- and off-task thoughts. All items were measured on a 5-point Likert scale ranging from 1 (not at all) to 5 (very much so), unless mentioned otherwise.

On-task thoughts were measured with a self-developed six-item scale. A sample item is: "I found it easy to concentrate on the task" (for all items see Appendix). Cronbach's alpha was .87. 
Off-task thoughts: We measured self-evaluative off-task thoughts with six items from Sarason et al.'s (1986) Cognitive Interference Questionnaire (CIQ), which is a standard and widely used instrument for the assessment of intrusive thoughts (for exact items see Appendix). General mind-wandering was measured with two items: One from the original CIQ questionnaire, "To what degree did your mind wander off the task?" and a self-developed item, "While playing the game did you have any other things going through your mind besides thoughts about the game (e.g. did you think about what you will be doing later today, your friends or family, something you found funny, some homework or assignment you should work on?)" Both scales had adequate internal consistencies ( $\alpha=.74$ and $\alpha=.75$, respectively).

Confirmatory factor analyses: As our on-task thoughts scale was newly developed it was important to ascertain whether the scale had discriminant validity when compared to the off-task thoughts scales. We conducted three confirmatory factor analyses in EQS, using the maximum likelihood estimation, in which all items were only allowed to load on their intended factor, factors were allowed to correlate among each other, and errors were not allowed to correlate. A three-factor model distinguishing between on-task thoughts, self-evaluative off-task thoughts, and general mind-wandering showed good fit, $\chi^{2}(74, N=78)=95.77, p=.045, \mathrm{CFI}=.95, \mathrm{SRMR}=.07$, RMSEA $=.06$. A two-factor model distinguishing between on-task thoughts, and combining self-evaluative off-task thoughts with general mindwandering fit the data significantly worse than the three-factor model, $\Delta \chi^{2}(2)$ $=30.41, p<.001, \chi^{2}(76, N=78)=126.18, p<.001, \mathrm{CFI}=.88, \mathrm{SRMR}=.08$, RMSEA $=.09$. Similarly, a single-factor model combining on-task with off-task thoughts fit the data significantly worse than the three-factor model, $\Delta \chi^{2}(3)=53.30, p<.001, \chi^{2}(77, N=78)=149.07, p<.001, \mathrm{CFI}=.83, \mathrm{SRMR}$ $=.09$, RMSEA $=.11$. The average variance extracted (AVE) of the on-task thoughts, self-evaluative off-task thoughts, and general mind-wandering scales was $.56, .34$, and .65 , respectively. The composite reliability (CR) of the on-task thoughts, self-evaluative off-task thoughts, and general mindwandering scales was $.83, .70$, and .68 , respectively. As expected, on-task thoughts were highly and negatively correlated with self-evaluative off-task thoughts (-.71) and with general mind-wandering (-.64). Self-evaluative offtask thoughts were highly correlated with general mind-wandering (.65). Overall, the current results indicate acceptable measurement properties of the scales used.

Control Variables. We controlled for gaming experience because more experienced participants likely find it easier to stay task-focused than less experienced participants, which in turn could affect their on-task and off-task thoughts ratings. Gaming experience was measured with three items: "How often do you play computer games in general?" $(1=$ almost never to $5=$ every 
day), "Have you ever played a similar game before (e.g. Lemmings?)" $(0=$ yes, $1=n o$ ), and "How experienced do you consider yourself in playing these kinds of games?" $(1=$ beginner to $5=$ expert $)$. In addition, in all analyses we also controlled for sex.

Manipulation Check. As a manipulation check at the end of the study we asked all participants if they could recall what strategy to handle errors we had recommended to them at the beginning of the study. Participants could choose among four options: error management, error prevention, another strategy, and "not sure". Three participants who could not properly recall which text they had read were removed from all subsequent analyses.

\section{Results}

Table 1 presents the means, standard deviations, and correlations for the experimental variables. Because making errors was integral to our theorising we wanted to establish whether participants in both conditions indeed made errors during the game. For this purpose, we calculated the number of all penguins lost as well as the number of level repetitions, both of which can be seen as consequences of errors that were made. The total number of penguins lost ranged between 21 and 147 with a mean of $72.21(S D=27.10)$. The mean number of level repetitions was $2.63(S D=1.79)$. There were no differences between conditions on the total number of penguins lost, $F(1,73)=0.36, p=$ $.552, \eta^{2} \mathrm{p}=0.01$, Cohen's $d=-0.14$, or on the number of level repetitions, $F(1$, 76) $=0.20, \mathrm{p}=.897, \eta_{\mathrm{p}}^{2}=0.00$, Cohen's $d=-0.03$. Based on these results we can conclude that the task induced errors independently of condition.

On-Task Thoughts. We predicted that participants in the error management condition would have more on-task thoughts than participants in the error prevention condition. An ANCOVA showed that participants in the error management condition $(M=4.34 ; S D=0.62)$ reported having more on-task thoughts than participants in the error prevention condition $(M=$ 4.04; $S D=0.66), F(1,72)=4.54, p=.037, \eta_{\mathrm{p}}^{2}=0.06$, Cohen's $d=0.48$ (Hypothesis 1 supported). There was also a significant effect of playing games in general, $F(1,72)=5.11, p=.027, \eta_{\mathrm{p}}^{2}=0.07$.

Off-Task Thoughts. A MANCOVA (using Pillai's trace) showed no significant effect of error instructions on off-task thoughts, $V=0.03, F(2,71)=$ $1.20, p=.307$ (Cohen's $d$ s were -0.35 and -0.15 for self-evaluative and general mind-wandering off-task thoughts, respectively). Thus, participants who received error management instructions did not report fewer off-task thoughts than those who received error prevention instructions (Hypothesis 2 not supported). 


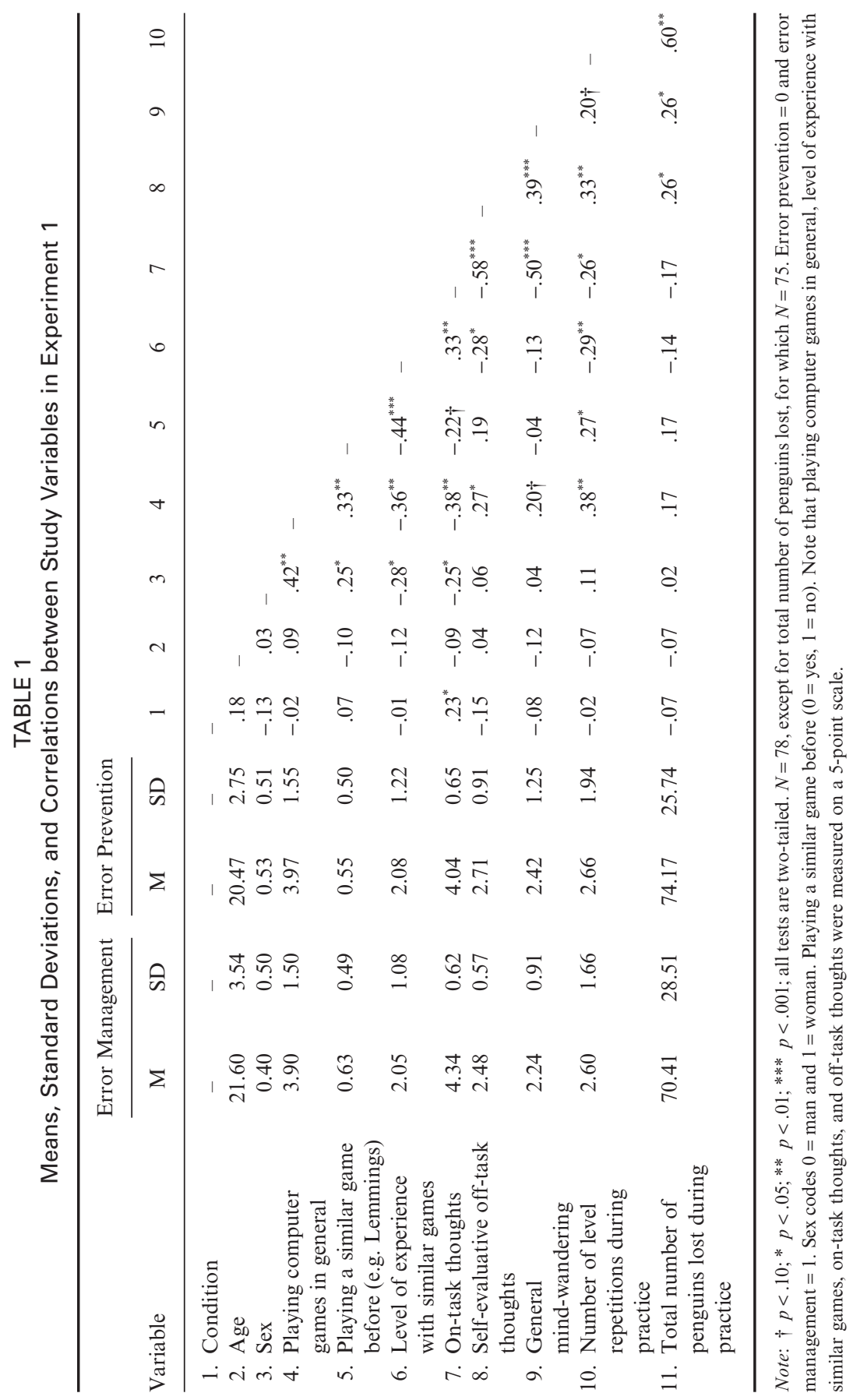




\section{Discussion}

As expected, Experiment 1 showed that participants who received error management instructions found it easier to focus on the task than participants who received error prevention instructions. No effect of error instructions on off-task thoughts was found. It is possible that because the task was highly engaging there was little opportunity for off-task thoughts. This limitation is addressed in Experiment 2 by incorporating short breaks (cf. Kanfer, Ackerman, Murtha, Dugdale, \& Nelson, 1994) allowing participants to "wander off" the task.

In Experiment 1 we selected a task during which-as is the case in real life - it was not always obvious if and when an error was made. Although using such a task increases the ecological validity of the experiment, it has the potential limitation that participants may have been unaware of an error if it did not immediately result in losing a penguin. This limitation is addressed in Experiment 2 by selecting a task that provides an error message immediately after an error occurs.

\section{EXPERIMENT 2}

\section{Method}

Participants and Design. The final sample consisted of 76 participants; all but one were students at the university of the first author ( 42 females and 34 males; $M_{\text {age }}=21.39, S D=3.22$ ). Participants were recruited in the same way as for Experiment 1. Participants were quasi-randomly (i.e. equal proportion of males and females per condition) assigned to either the error management (20 females and 19 males) or the error prevention condition (22 females and 15 males). Four participants in the initial sample were removed from all analyses: one participant was not reading the instructions/questions and three participants indicated to the experimenter during and after the experiment that they were not proficient with computers. All participants received $€ 10$ for taking part in the research.

Procedure. Experiment 2 lasted approximately 90 minutes. Participants were seated in separate cubicles and were told that most instructions and all questions would appear on the computer screen. In order to increase the chance that participants would read the manipulation carefully we presented them with a cover story. Namely, we told participants that we would first collect data on their personality, after which we would suggest to them a strategy that fits with their personality to use during the subsequent task.

Simulation task: The task used was a free-source PC simulation called Train Dispatcher 2.0๑ (Signal Computer Consultants, 1997). In this simulation, a participant acts as a train dispatcher who guides trains from their 
entry to their exit location. A useful feature of the simulation was that if participants made an error they would immediately receive an error message (however, no error message was shown if participants sent a train to the wrong end destination, but only if participants were using the wrong signals and switches or trying to do something impossible). Participants could choose to speed up or slow down the simulation with a time multiplier. Faster speeds made the simulation more challenging and added extra points to the final score. The software automatically calculated the scores.

Task familiarisation: All participants were presented with identical basic instructions outlining how to use the signals and switches, start and stop the simulation, zoom in and out, and check train destinations. Participants were then informed that the main goal was to guide as many trains as possible to their correct end destinations as quickly as possible. After reading the instructions participants had an opportunity to familiarise themselves with the simulation for five minutes by guiding a train from its start to its end destination. The experimenter gave participants a handout with detailed instructions on what the train number was, where the train would appear and where the train should be directed to. In addition, participants received a handout with the general task instructions presented before the familiarisation trial. Participants worked on this first task for 5 minutes. If within 5 minutes participants did not manage to guide the train to its end destination the experimenter showed them how the task could be successfully completed.

Manipulation error approach: After the task familiarisation stage participants saw a screen indicating that based on their personality they should follow the strategy mentioned next, which was the error approach manipulation. The manipulations were identical to those in Experiment 1 with the exception of a few minor word changes to improve wording and connect the manipulation to the cover story.

Manipulation check: After reading the manipulation text we asked participants to write a short summary (maximum of 200 words) explaining the strategy mentioned in the text they had previously read. The summaries were coded by two independent coders, knowledgeable on the error approach literature (both coders belonged to a research group investigating errors that the first and second authors are a part of), but blind to experimental condition. The two coders had 100 per cent agreement on the type of strategy described by participants (management or prevention). For all participants, the summaries, as coded by the coders, matched the condition that participants were assigned to, indicating that all participants had read the manipulation.

Practice: After the manipulations were administered, all participants began their task practice, consisting of two 10-minute trials separated by a 2-minute break. During the practice all participants were provided with a 
train schedule outlining when trains would appear on the screen (in simulation time) and what their start and end destination was, as well as a copy of the full simulation manual (including all available information on the simulation, not only the basic information provided during the familiarisation trial).

On- and off-task thoughts: After the completion of the practice trials all participants had a 2-minute break before filling in the measures of on- and off-task thoughts, which were identical to the measures used in Experiment 1. All ratings were made on a 7-point Likert scale ranging from 1 (not at all) to 7 (very much so). Cronbach's alphas were $.89, .75$, and .83 for on-task thoughts, self-evaluative off-task thoughts, and general mind-wandering, respectively.

Performance: Following the on-task and off-task thoughts measures, participants completed two 10-minute performance trials, which were described as an opportunity to show what participants had learned (cf. Keith \& Frese, 2005). Participants were instructed to guide as many trains as possible to their correct end destinations. The first 10-minute performance trial measured analogical transfer. The task in the analogical transfer trial had a level of difficulty comparable to that in the practice phase and involved similar procedures. The final score of the first performance trial was used as the measure of analogical transfer performance.

After a 2-minute break participants worked on the second 10-minute performance trial, measuring adaptive transfer. The task in the adaptive transfer trial was more difficult than both the practice and the analogical transfer trials. The task included a more complex track territory with a higher number of tracks and trains, as well as new instruments and rules, which required participants to update their previously created mental model of the task (i.e. the novel elements considerably changed how the simulation behaved as compared to the previous sessions; see Bell \& Kozlowski, 2008; Keith \& Frese, 2005). The final score of the second performance trial was used as a measure of adaptive transfer performance. To provide additional motivation to perform during the performance trials, participants were told that the person with the highest score would receive $€ 50$.

Control Variables. None of the participants indicated having prior experience with the specific simulation task used ("Have you ever played this simulation before?", yes vs. no). Prior experience with similar tasks was measured with one item: "Do you have previous experience with similar tasks?" ( 1 = not at all, 7 = a lot of experience $)$. In addition, we included an item measuring participants' agreement with the cover story, "Did you think the strategy we suggested for you fits you?" $(1=$ not at all, $7=$ completely). Finally, in all analyses we controlled for sex. 


\section{Results}

Table 2 presents the means, standard deviations, and correlations. To test whether the task was successful at inducing errors, we measured the number of errors by calculating the number of error messages $(M=41.30 ; S D=28.48)$ participants saw during the two 10-minute practice trials. No differences between conditions were found during practice trial $1, F(1,72)=2.25, p=$ $.138, \eta_{\mathrm{p}}^{2}=0.03$, Cohen's $d=0.35$, or practice trial $2, F(1,72)=0.288, p=.589$, $\eta_{\mathrm{p}}^{2}=0.00$, Cohen's $d=0.13$. Also, the total number of error messages received during the whole practice phase (practice trial $1+2$ ) did not vary significantly between conditions, $F(1,72)=1.14, p=.29, \eta_{\mathrm{p}}^{2}=0.02$, Cohen's $d=0.25$. Based on these results we conclude that participants in the two conditions made a similar number of errors during the practice phase.

Main Effects. On-task thoughts: An ANCOVA showed a main effect of condition on on-task thoughts, $F(1,71)=4.71, p=.033, \eta^{2}=0.06$, Cohen's $d=-0.50$. Consistent with Experiment 1, error management $(M=5.81 ; S D$ $=1.05)$ resulted in more on-task thoughts than did error prevention $(M=5.21$; $S D=1.07$; Hypothesis 1 supported). There was also a significant effect of perceived fit of the suggested strategy with one's personality on on-task thoughts, $F(1,72)=6.26, p=.015, \eta_{\mathrm{p}}^{2}=0.08$, Cohen's $d=0.57$.

Off-task thoughts: A MANCOVA (using Pillai's trace) showed no significant effect for condition, $V=0.06, F(2,70)=2.10, p=.131, \eta_{\mathrm{p}}^{2}=0.06$ (Cohen's $d$ self-evaluative off-task thoughts $=-0.43$; Cohen's $d$ general mindwandering off-task thoughts $=0.19$ ). There was a significant effect of the covariate perceived fit of the suggested strategy with one's personality on off-task thoughts, $V=0.18, F(2,70)=7.66, p=.001, \eta_{\mathrm{p}}^{2}=0.18$, driven by the variable's effect on general mind-wandering, $F(1,71)=15.13, p<.001, \eta^{2}{ }_{\mathrm{p}}=$ 0.18 . The current findings indicate that participants who received error management instructions did not have fewer off-task thoughts compared to participants who received error prevention instructions (Hypothesis 2 not supported).

Practice performance: An ANCOVA showed no effect of condition on the first 10-minute practice score, $F(1,69)=0.92, p=.341, \eta^{2}{ }_{p}=0.01$, Cohen's $d$ $=-0.22$. However, error management participants performed significantly better than error prevention participants $(M=0.27 ; S D=1.03$ vs. $M=-0.28$; $S D=0.90$, standardised scores) on the second 10-minute practice trial, $F(1$, 71) $=5.16, p=.027, \eta_{\mathrm{p}}^{2}=0.07$, Cohen's $d=-0.52$.

Analogical transfer performance: An ANCOVA showed a main effect of condition on analogical transfer performance, $F(1,71)=7.69, p=.007, \eta^{2}{ }_{\mathrm{p}}=$ 0.10 , Cohen's $d=-0.64$. Supporting Hypothesis 3, error management resulted in better analogical transfer performance than error prevention $(M$ $=0.30 ; S D=1.17$ vs. $M=-0.32 ; S D=0.65$, standardised scores). 


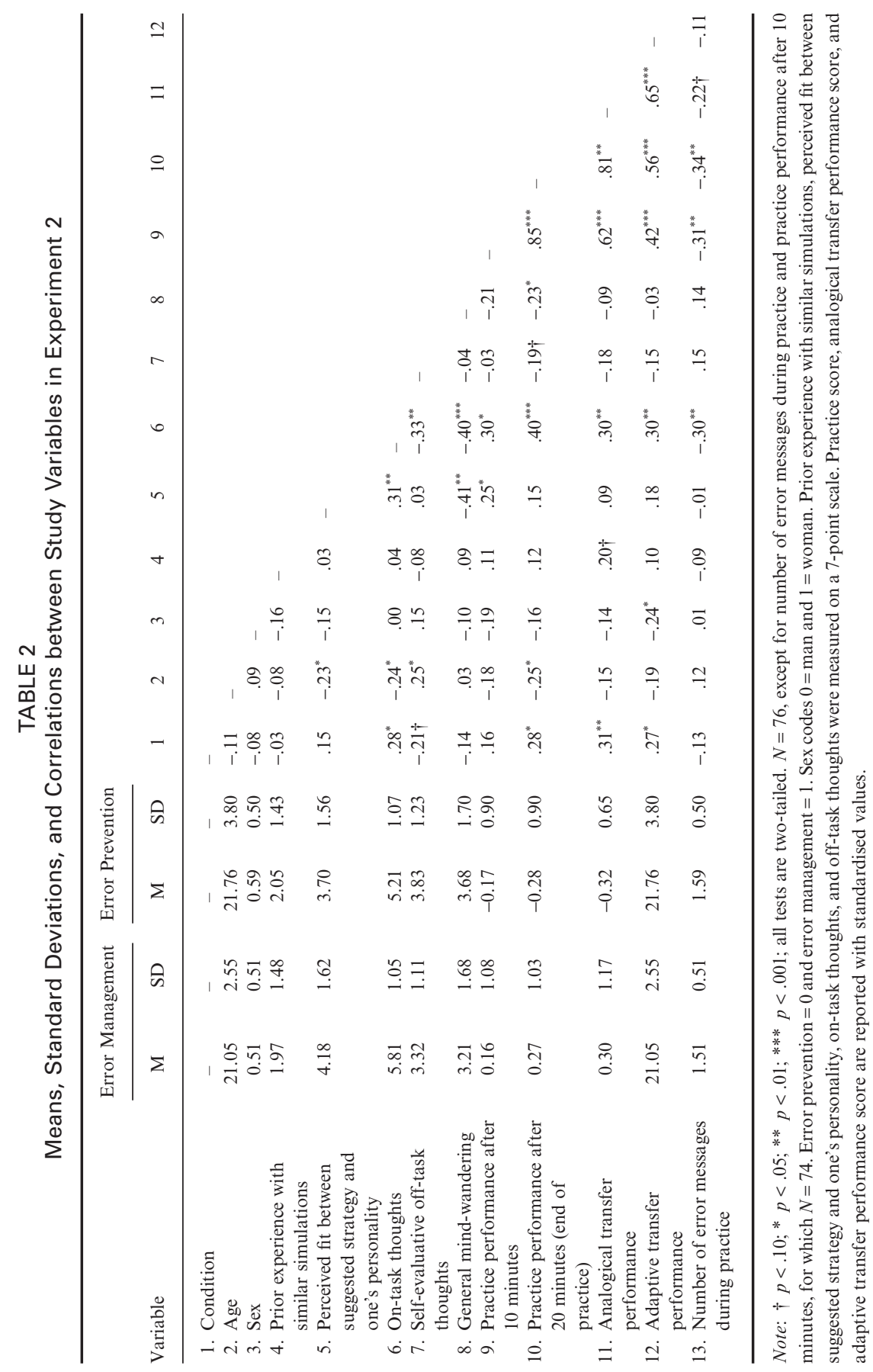


Adaptive transfer performance: An ANCOVA showed a main effect of condition on adaptive transfer performance, $F(1,71)=4.53, p=.037, \eta^{2}{ }_{\mathrm{p}}=$ 0.06 , Cohen's $d=-0.49$, with error management resulting in better adaptive transfer performance than error prevention $(M=0.26 ; S D=1.14$ vs. $M=$ $-0.27 ; S D=0.74$, standardised scores; Hypothesis 4 supported).

Mediation Analyses. To conduct the mediation analyses we used Preacher and Hayes' (2008) SPSS multiple mediation script with a 5,000 bootstrap resample. The script allows multiple mediators to be entered simultaneously and automatically calculates bias-corrected and accelerated confidence intervals. All mediation analyses included the control variables.

Analogical transfer performance: The total indirect effect of all proposed mediators assessed simultaneously was not significant, $B=0.07, S E=0.04$, $95 \%$ CI $[-0.004,0.18]$, which could be expected based on the lack of correlation between the off-task thoughts measures and error approach or transfer performance. When examining the mediators individually, results indicated that only on-task thoughts mediated the effect of error approach on analogical transfer performance, $B=0.06, S E=0.04,95 \%$ CI [0.01, 0.18]. Hypothesis 5 a was supported. Self-evaluative off-task thoughts, $B=0.004, S E=0.03$, $95 \%$ CI $[-0.05,0.08]$, and general off-task thoughts, $B=0.0003, S E=0.02$, $95 \%$ CI [ $-0.04,0.04]$, were not mediators (Hypothesis 6a not supported).

Adaptive transfer performance: The total indirect effect of all proposed mediators tested simultaneously was not significant, $B=0.05, S E=0.05,95 \%$ CI $[-0.05,0.15]$. Examination of individual mediators, however, showed that on-task thoughts mediated the effect of error approach on adaptive transfer performance, $B=0.06, S E=0.04,95 \% \mathrm{CI}[0.01,0.19]$, supporting Hypothesis 5b. Self-evaluative off-task thoughts, $B=-0.003, S E=0.04,95 \%$ CI [ -0.13 , $0.05]$, and general off-task thoughts, $B=-0.01, S E=0.03,95 \%$ CI $[-0.09$, 0.02 ], were not mediators (Hypothesis $6 \mathrm{~b}$ not supported).

\section{Discussion}

Experiment 2 replicated the finding of Experiment 1 that error management results in more on-task thoughts than does error prevention. In addition, confirming our predictions, error management resulted in both better analogical and adaptive transfer performance. Interestingly, we found a main effect of error instructions on practice performance that was not expected. Separate tests of performance in the first and second part of the practice task showed that participants in both conditions performed similarly during the first 10 minutes of practice while performance differences became apparent during the second 10 minutes. A possible explanation for this finding is that 10 minutes were enough for participants to develop task-relevant declarative knowledge - the knowledge of rules and skills necessary to do a task (Kanfer 
\& Ackerman, 1989) resulting in improved scores in the error management condition. Finally, our analyses showed that on-task thoughts mediated the effects of error instructions on both analogical and adaptive transfer performance, whereas off-task thoughts did not.

\section{GENERAL DISCUSSION}

The current studies extend prior knowledge of cognition as a mediator of the effects of error approach on performance (e.g. Keith \& Frese, 2005). Overall, our findings show that error management instructions promote both analogical and adaptive transfer performance by activating more on-task thoughts than error prevention instructions. The results indicate that error management enhances performance not by minimising task-undirected (offtask) thoughts, but by making it more likely that people focus on the task at hand.

\section{Theoretical and Methodological Implications}

Our findings both replicate and extend previous research (e.g. Bell \& Kozlowski, 2008; Frese et al., 1991; Heimbeck et al., 2003; Keith \& Frese, 2005, 2008; Nordstrom et al., 1998). We build on Keith and Frese's (2005) idea that error approach affects performance by influencing cognitive functioning (Kanfer \& Ackerman, 1989) by a more positive reappraisal of errors under error management instructions than under error prevention instructions (also see Richards \& Gross, 2000). We extend Keith and Frese's research by showing that on-task thoughts mediate the effect of error approach on performance, while off-task thoughts do not.

According to earlier reasoning, stress and worry caused by error occurrence negatively affect cognition, directing thoughts away from the task (e.g. Frese et al., 1991; Heimbeck et al., 2003; Keith \& Frese, 2005; Nordstrom et al., 1998). In line with this reasoning, we expected that error prevention instructions would cultivate off-task thoughts, and harm performance. Instead, our results suggest that error management leads to more on-task thoughts, while off-task thoughts are not affected by error approach. The important implication of these findings is that subsequent theorising should focus less on the potential negative effects of error prevention on cognition (more off-task thoughts), and more on the positive effects of error management on on-task thoughts. Put differently, the mechanisms explaining the effects of error approach on performance may be positive, rather than negative.

We extend the work of Keith and Frese (2005) and Nordstrom et al. (1998) by using manipulations that vary on error approach only. Specifically, we kept the manipulation length similar in both conditions, framed both error management and error prevention strategies positively, and informed 
participants in both conditions that the respective strategy was good for learning and performance. In addition, the manipulations we used are easier and faster to administer, making their application to real-life contexts less complex. With this more parsimonious error approach manipulation, we nonetheless replicate prior performance effects (Keith \& Frese, 2008), attesting to the robustness of the superiority of error management over error prevention.

We carefully selected the tasks we used in the current studies so as to ensure that they would not favor either management or prevention. In addition, in earlier research, the error management group typically received error-based practice while the error prevention group received errorless practice (Bell \& Kozlowski, 2008; Heimbeck et al., 2003; Keith \& Frese, 2008). In the current research, in contrast, participants in both the error management and the error prevention condition were offered the opportunity for exploratory task practice. Our results indicate that the usefulness of errors during practice depends on whether people receive error management or error prevention instructions. Even though the error management and the error prevention groups made a similar number of errors during practice, the error management group was better able to stay task-focused and learn from the errors resulting in better analogical and adaptive transfer performance. Accordingly, the combination of making errors and being able to reappraise them as learning opportunities seems to make error management more effective than error prevention (also see Heimbeck et al., 2003).

\section{Limitations}

A possible limitation is related to the measurement of on- and off-task thoughts. Like others (e.g. Kanfer \& Ackerman, 1989; Keith \& Frese, 2005), we measured cognition with self-report items. However, other measures may be better suited to capture on- and off-task thoughts. Future work could attempt to address this issue by measuring on- and off-task thoughts by asking participants to say out loud what they are thinking about during the practice itself. Nonetheless, the speaking out loud procedure has its own limitations: people are not always capable of verbalising their thoughts and speaking may take attention away from the task itself, resulting in more off-task thoughts.

Second, it can be argued that our measure of on-task thoughts shows some similarities with the concept of emotion control (Keith \& Frese, 2005). On-task thoughts and self-regulation, however, are theoretically distinct. Emotion control refers to the self-regulation part of Kanfer and Ackerman's (1989) cognitive resource allocation model, reflecting changes in resource allocation. Our measure, in contrast, represents on-task thoughts not changes in resource allocation. Nevertheless, future work should include 
both measures of on-task thoughts and self-regulation simultaneously so that their effects can be compared.

Third, another potential limitation of the current research is the measurement of both analogical and adaptive transfer performance after the practice phase. Although the current procedure was purposefully selected because it has been validated in previous research (see Bell \& Kozlowski, 2008), we cannot rule out the possibility that it induced habituation in participants. This issue should be addressed in future research by forming separate performance groups: one group with only an analogical transfer performance trial after the practice phase and a second group with only an adaptive transfer performance trial after the practice phase.

Finally, the effects of error approach over time should be tested using a longitudinal design. Based on existing research, it is unclear how long performance benefits last. Although some previous work has investigated the effects of error approach on performance a week after training (e.g. Heimbeck et al., 2003), to the best of our knowledge no experimental study has tested performance on multiple occasions over a longer term.

\section{Practical Implications and Future Research}

A potentially important practical implication of our findings is that even brief error management instructions improve people's ability to stay task-focused and to manage whatever goes wrong. Error management may thus increase the efficiency of job-training and benefit task performance. The manipulations used in both studies were only three paragraphs long, yet the participants who received error management instructions performed better than the participants who received error prevention instructions. Incorporating errorframing instructions while instructing people to do a new task in the workplace (or elsewhere) is simple, easy, and efficient, and the increases in performance can be beneficial to both individuals and organisations.

To test whether the effects of error management instructions on on-task thoughts and performance can be generalised to the workplace, our findings should be tested in an organisational setting, using a quasi-experimental design. Previous research by Van Dyck and colleagues (2005) provided evidence that organisational error management culture was positively linked to firm goal achievement and profitability. Note that the research by Van Dyck and colleagues involved overall performance based on how companies performed on both new and established tasks. We can speculate that error management instructions may be beneficial to performance not only on new, but also on well-learned, tasks that employees perform. Such a suggestion, however, should be regarded as speculative. More research on the effects of error approach instructions for both novel and well-learned tasks is warranted. 
An interesting question that remains to be answered is whether our findings can be generalised to tasks with different levels of difficulty within organisations. While some tasks are simple and routine, others are complex and difficult to learn. Experimental non-field research indicates that the benefits of error management for learning and performance mainly become apparent for difficult but not for simple tasks (Frese et al., 1991). To the extent that error management can help people stay task-focused, as indicated by our results, it may also be beneficial to long-term performance even on simple tasks. Future research should investigate this possibility.

Another interesting question is whether error management is always superior to prevention. What we argue is not that making errors per se is good, but rather that error management allows people to handle the existing errors so that their negative consequences are minimised. Error management differentiates the error from its consequences and attempts to control the latter, whereas error prevention tries to evade the negative consequences by eradicating errors per se (Hofmann \& Frese, 2011). Both prevention and management are important to organisations. Errors, however, cannot be fully eradicated (Reason, 1990) and it is when prevention fails that error management becomes vital for trapping errors and minimising their negative consequences.

Finally, the current findings suggest that being able to focus on the task is what makes error management particularly effective in improving performance. Although more research applying error instructions in real organisations is necessary, our findings indicate that error management can be beneficial not only because it helps people with tasks similar to those previously practiced, but also because it teaches people how to successfully master errors and handle new problems. In line with Bell and Kozlowski (2008), we argue that it is not possible to prepare employees for everything that can potentially go wrong. Organisational environments are becoming increasingly less predictable and more complex, necessitating that people become more adaptive, flexible, and efficient. In addition, staying task-focused with all the present-day distractions is progressively more challenging. We suggest that error management can aid organisations in producing the type of resilient employee who can stay task-focused and successfully adapt quickly to new situations and problems.

\section{REFERENCES}

Bell, B.S., \& Kozlowski, S.W.J. (2008). Active learning: Effects of core training design elements on self-regulatory processes, learning, and adaptability. Journal of Applied Psychology, 93, 296-316. doi: 10.1037/0021-9010.93.2.296

Debowski, S., Wood, R.E., \& Bandura, A. (2001). Impact of guided exploration on self-regulatory mechanisms and information acquisition through electronic 
search. Journal of Applied Psychology, 86, 1129-1141. doi: 10.1037/00219010.86.6.1129

Dormann, T., \& Frese, M. (1994). Error training: Replication and the function of exploratory behavior. International Journal of Human-Computer Interaction, 6, 365-372. doi: 10.1080/10447319409526101

Ford, J.K., Smith, E.M., Weissbein, D.A., Gully, S.M., \& Salas, E. (1998). Relationships of goal orientation, metacognitive activity, and practice strategies with learning outcomes and transfer. Journal of Applied Psychology, 83, 218-233. doi: $10.1037 / 0021-9010.83 .2 .218$

Frese, M., Brodbeck, F., Heinbokel, T., Mooser, C., Schleiffenbaum, E., \& Thiemann, P. (1991). Errors in training computer skills: On the positive function of errors. Human-Computer Interaction, 6, 77-93. doi: 10.1207/s15327051hci0601 -3

Frese, M., \& Zapf, D. (1994). Action as the core of work psychology: A German approach. In H.C. Triandis, M.D. Dunette, \& L.M. Hough (Eds.), Handbook of industrial and organizational psychology (Vol. 4, pp. 271-340). Palo Alto, CA: Consulting Psychologists Press.

Heimbeck, D., Frese, M., Sonnentag S., \& Keith, N. (2003). Integrating errors into the training process: The function of error management instructions and the role of goal orientation. Personnel Psychology, 56, 333-361. doi: 10.1111/j.17446570.2003.tb00153.x

Hofmann, D.A., \& Frese, M. (Eds.) (2011). Errors in organizations. New York: Routledge.

Ivancic, K., \& Hesketh, B. (1995/1996). Making the best of errors during training. Training Research Journal, 1, 103-125.

Ivancic, K., \& Hesketh, B. (2000). Learning from error in a driving simulation: Effects of driving skill and self-confidence. Ergonomics, 43, 1966-1984. doi: 10.1080/ 00140130050201427

Joung, W., Hesketh, B., \& Neal, A. (2006). Using "war stories" to train for adaptive performance: Is it better to learn from error than success? Applied Psychology: An International Review, 55, 282-302. doi: 10.1111/j.1464-0597.2006.00244.x

Kanfer, R., \& Ackerman, P.L. (1989). Motivation and cognitive abilities: An integrative/aptitude-treatment interaction approach to skill acquisition. Journal of Applied Psychology, 74, 657-690. doi: 10.1037/0021-9010.74.4.657

Kanfer, R., Ackerman, P.L., Murtha, T.C., Dugdale, B., \& Nelson, L. (1994). Goal setting, conditions of practice, and task performance: A resource allocation perspective. Journal of Applied Psychology, 79, 826-835. doi: 10.1037//00219010.79.6.826

Keith, N., \& Frese, M. (2005). Self-regulation in error management training: Emotion control and metacognitive activity as mediators of performance effects. Journal of Applied Psychology, 90, 677-691. doi: 10.1037/0021-9010.90.4.677

Keith, N., \& Frese, M. (2008). Effectiveness of error management training: A metaanalysis. Journal of Applied Psychology, 93, 59-69. doi: 10.1037/0021-9010.93 .1 .59

Lee, F.K., Sheldon, K.M., \& Turban, D.B. (2003). Personality and the goal-striving process: The influence of achievement goal patterns, goal level, and mental focus 
on performance and enjoyment. Journal of Applied Psychology, 88, 256-265. doi: 10.1037/0021-9010.88.2.256

Nordstrom, C.R., Wendland, D., \& Williams, K.B. (1998). "To err is human": An examination of the effectiveness of error management training. Journal of Business and Psychology, 12, 269-282. doi: 10.1023/A:1025019212263

Preacher, K.J., \& Hayes, A.F. (2008). Asymptotic and resampling strategies for assessing and comparing indirect effects in multiple mediator models. Behavior Research Methods, 40, 879-891. doi: 10.3758/BRM.40.3.879

Reason, J. (1990). Human error. New York: Cambridge University Press.

Reason, J. (1997). Managing the risks of organizational accidents. Aldershot: Ashgate.

Richards, J.M., \& Gross, J.J. (2000). Emotion regulation and memory: The cognitive costs of keeping one's cool. Journal of Personality and Social Psychology, 79, 410-424. doi: 10.1037/0022-3514.79.3.410

Ruhnke, I. (2010). Pingus@ $($ Retrieved from: http://pingus.seul.org/welcome.html

Sarason, I.G., Sarason, B.R., Keefe, D.E., Hayes, B.E., \& Shearin, E.N. (1986). Cognitive interference: Situational determinants and traitlike characteristics. Journal of Personality and Social Psychology, 51, 215-226. doi: 10.1037/00223514.51.1.215

Signal Computer Consultants (1997). Train Dispatcher (Version 2.0). Retrieved from: http://www.signalcc.com/train2/td2freeware.html

Sitkin, S.B. (1992). Learning through failure: The strategy of small losses. Research in Organizational Behavior, 14, 231-266.

Smallwood, J., Obonsawin, M., \& Heim, D. (2003). Task-unrelated thought: The role of distributed processing. Consciousness \& Cognition, 12, 169-189. doi: 10.1016/ S1053-8100(02)00003-X

Smallwood, J., Riby, L, Heim, D., \& Davies, J.B. (2006). Encoding during the attentional lapse: Accuracy of encoding during the semantic sustained attention to response task. Consciousness \& Cognition, 15, 218-231. doi: 10.1016/ j.concog.2005.03.003

Van Der Linden, D., Sonnentag, S., Frese, M., \& Van Dyck, C. (2001). Exploration strategies, performance, and error consequences when learning a complex computer task. Behavior and Information Technology, 20, 189-198. doi: 10.1080/ 01449290110047990

Van Dyck, C., Frese, M., Baer, M., \& Sonnentag, S. (2005). Organizational error management culture and its impact on performance: A two-study replication. Journal of Applied Psychology, 90, 1228-1240. doi: 10.1037/0021-9010.90.6.1228

Wood, R.E., Kakebeeke, B.M., Debowski, S., \& Frese, M. (2000). The impact of enactive exploration on intrinsic motivation, strategy, and performance in electronic search. Applied Psychology: An International Review, 49, 263-283. doi: 10.1111/1464-0597.00014

Yee, P.L., Hsieh-Yee, I., Pierce, G.R., Grome, R.E., \& Schantz, L.H. (2004). Self-evaluative intrusive thoughts impede successful searching on the Internet. Computers in Human Behavior, 20, 85-101. doi: 10.1016/S0747-5632(03)00042-6 


\section{APPENDIX: ITEMS USED IN EXPERIMENTS 1 AND 2}

\section{On-task thoughts items (self-developed, based on Kanfer \& Ackerman, 1989 and Keith \& Frese, 2005)}

I found it easy to concentrate on the task.

I found it easy to keep thinking about what I was supposed to do.

My mind was busy with solving the task.

It was easy to get over a problem and get back to the task.

I could keep my mind on the task.

It was easy to concentrate on what I was doing.

\section{Off-task thought items (adapted from Sarason et al., 1986)}

Self-evaluative off-task thoughts items (based on the distinction of off-task thoughts by Yee, Hsieh-Yee, Pierce, Grome, \& Schantz (2004))

I thought about how poorly I was doing.

I thought about how I should work more carefully.

I thought about my level of ability.

I thought about how I would feel if I were told how I performed.

I thought about how often I got confused.

I thought about what the experimenter would think of me.

General mind-wandering (adapted from Sarason et al., 1986)

To what degree did your mind wonder off the task?

While playing the game did you have any other things going through your mind besides thoughts about the game (e.g. did you think about what you would be doing later today, your friends or family, something you found funny, some homework or assignment you should work on)? (Self-developed item combining multiple items from the original Sarason et al., 1986, scale.)

\section{Control Variables Experiment 1}

How often do you play computer games in general? $(1=$ almost never to $5=$ every day)

Have you ever played a similar game before (e.g. Lemmings?) $(0=$ yes, $1=n o)$

How experienced do you consider yourself in playing these kinds of games? $(1=$ beginner to 5 = expert $)$

What is your sex? $(0=$ man, $1=$ woman $)$ 


\section{Control Variables Experiment 2}

Have you ever played this simulation task before? (yes vs. no).

Do you have previous experience with similar tasks? $(1=$ not at all, $7=a$ lot of experience).

Did you think the strategy we suggested for you fits you? $(1=$ not at all, 7 = completely).

What is your sex? $(0=$ man, $1=$ woman $)$

\section{Manipulation Check Experiment 1}

Which strategy did we recommend you?

"To err is human", "Errors really are not that bad", and "Try to learn as much as you can from your errors!"

"Better safe than sorry", "Mistakes can be prevented through diligence", and "It is crucial to work faultlessly!"

"Another strategy"

"Not sure"

\section{Manipulation Check Experiment 2}

Make a short summary (minimum 50, maximum 200 words) of the strategy you received and mention its most important parts.

Note: The on-task and off-task thoughts items were presented in random order during both experiments. In Experiment 1 the response scale ranged from 1 (not at all) to 5 (very much so). In Experiment 2 the response scale ranged from 1 (not at all) to 7 (very much so). 